\title{
Microwave-assisted rapid preparation of hollow carbon nanospheres@TiN nanoparticles for lithium-sulfur batteries
}

\author{
Jianxin Tu ${ }^{\mathrm{a}, \mathrm{b}}$, HeJun Li ${ }^{\mathrm{a}}$, JiZhao Zou ${ }^{\mathrm{b}}$, ShaoZhong Zeng ${ }^{\mathrm{b}}$, Qi Zhang ${ }^{\mathrm{c}}$, \\ Liang $\mathrm{Yu}^{\mathrm{b}}$ and Xierong Zeng, ${ }^{\mathrm{a}, \mathrm{b}^{*}}$
}

\begin{abstract}
${ }^{\text {a }}$ State Key Laboratory of Solidification Processing, Carbon/Carbon Composites Research Center, Northwestern Polytechnical University, Xi'an 710072, China ${ }^{\mathrm{b}}$ Shenzhen Key Laboratory of Special Functional Materials \& Shenzhen Engineering Laboratory for Advance Technology of ceramics, College of Materials Science and Engineering, Shenzhen University, Shenzhen 518060, PR China

${ }^{\mathrm{c}}$ School of Aerospace, Transport, and Manufacturing, Cranfield University, Cranfield, UK
\end{abstract}

\begin{abstract}
:
Highly conductive titanium nitride (TiN) has a strong anchoring ability for lithium polysulfides (LiPSs). However, the complexity and high cost of fabrication limit their practical applications. Herein, a typical structure of hollow carbon nanospheres@TiN nanoparticles (HCNs@TiN) was designed and successfully synthesized via a microwave reduction method with the advantages of economy and efficiency. With unique structural and outstanding functional behavior, HCN@TiN-S hybrid electrodes display not only a high initial discharge capacity of $1097.8 \mathrm{~mA} \mathrm{~h}$ g- 1 at $0.1 \mathrm{C}$, but also excellent rate performance and cycling stability. After 200 cycles, a reversible capacity of $812.6 \mathrm{mAh} \mathrm{g}^{-1}$ is still retained, corresponding to the low decay rate of $0.13 \%$ per cycle of the initial capacity, which are much better than those of contrast HCNs-S electrodes.
\end{abstract}

Keywords: TiN; Hollow carbon nanospheres; Microwave reduction; Li-S batteries 
*Corresponding author. Tel.: +86 755 26537459;

E-mail: zengxier@szu.edu.cn; zoujizhao@szu.edu.cn

\section{Introduction}

To meet the growing demands for advanced energy storage system, Li-S batteries are considered as one of the most promising candidates for the next generation large-scale energy storage devices due to the extraordinary theoretical specific capacity and energy density of sulfur (1675 $\mathrm{mAh} \mathrm{g}^{-1}$ and $2600 \mathrm{Wh} \mathrm{kg}^{-1}$ or $\left.2800 \mathrm{Wh} \mathrm{L}^{-1}\right)$ [1]. However, the low electron conductivity of sulfur $\left(\sim 10^{-30} \mathrm{~S} \mathrm{~cm}^{-1}\right)$ and the dissolution of lithium polysulfides in organic electrolytes are two key obstacles, which hinder Li-S batteries commercialization process [2].

To counter these problems, an effective method is to combine sulfur nanoparticles with other conductive matrix materials [3]. Over the past few years, carbonaceous materials, such as carbon spheres [4-6], carbon nanotubes [7], porous carbon fibers [8], carbon nanoribbons [9] and graphene [10], have been widely introduced in Li-S batteries as sulfur host materials because of their intrinsic high conductivity, high porosity, high surface area, diversity in nanostructures and easy availability [11]. Among them, hollow carbon nanospheres are considered as very promising matrix materials due to their fully enclosed structure and large inner cavity [12-15]. It basically solves the problem of electrical insulation and the large volumetric expansion of sulfur. However, these nonpolar carbon based nanocomposites can only provide a weak physical adsorption to the LiPS. Once the polar polysulfides are dissolved in the organic electrolyte, the reutilization of sulfur becomes very difficult, which makes the sulfur/carbon composite cathodes still generally suffer from low cycle stability.

Except for encapsulating sulfur within the pores of carbon materials, the strong chemical interaction effect between LiPSs and the matrix has attracted more attention 
and interest from the researcher. Some of materials such as conductive polymers [16], metallic oxide [17-19], sulfides [20, 21] and hydroxides [22] have been combined with carbon materials as the sulfur host because of their strong chemical affinity with LiPSs. However, most of the above chemical adsorbents are semiconducting or even electronic insulating, which seriously impedes electron transportation within the cathode. Therefore, there will be an important value in introducing highly conductive polar materials into the sulfur electrode to alleviate the above issues.

Recently, early-transition-metal carbides or nitrides having high conductivity, a polar surface, electrocatalytic activity and low cost have been demonstrated as good electrode additives for improving the cycling stability of Li-S batteries [23,24]. Yu and coworkers presented a strategy that is decorating metal carbide nanoparticles $\left(\mathrm{W}_{2} \mathrm{C}, \mathrm{Mo}_{2} \mathrm{C}\right.$ and $\mathrm{TiC}$ ) as active sites on CNFs as the electrodes [25]. Electrochemical tests demonstrate that carbide nanoparticles could improve both the cycling stability and rate capability of Li-S batteries via the dual effect of providing adsorption and catalytic ability. In addition, transition metal nitrides have no less performance than carbides. In 2016, a mesoporous TiN as sulfur host for Li-S batteries was first reported by Goodenough and co-workers [26]. This porous TiN could confine and encapsulate LiPSs by both chemical interactions and physical adsorption. Furthermore, TiN has high electrical conductivity and excellent chemical stability. All these properties make the hybrid TiN-S an excellent cathode. Most recently, the reaction mechanism of polysulfide catalyzed by TiN has been investigated in detail by Ding et al [27]. The density functional theory (DFT) calculations revealed that the long-chain $\mathrm{Li}_{2} \mathrm{~S}_{8}$ tend to break down into two shorter chain segments due to the strong interaction of TiN. These efforts mentioned above indicated that TiN can be applied as ideal cathode material of Li-S batteries. 
However, synthesis method of TiN usually involved a thermal reaction via ammoniation at prolonged high-temperature [26-31]. This process has some shortcomings, such as high time and energy consumption, environmentally unfriendly reactants and high-cost, and thus, is not a practical method for fabricating engineering materials in the bulk form. Besides, out of consideration for the overall energy density of the electrode, it is inappropriate to overuse the TiN as the host material for sulfur due to its relative high density.

Taking the above discussion into consideration, in this study, we use a one-step route to fabricate poly(aniline-co-pyrrole)(PACP) copolymer hollow nanospheres by the polymerization of pyrrole and aniline in Triton X-100 solution. And an effective solgel and microwave reduction method was designed and used to obtain a typical structure of hollow carbon nanospheres@TiN nanoparticles (HCNs@TiN) as the the host materials for Li-S batteries.Particularly, PACP@TiO2 can be transformed into the HCNs@TiN composite completely at $1200 \mathrm{C}$ for only 10 min by microwave heating reduction under a N2 atmosphere. This method not only reduces the reaction temperature significantly through microwave heating but also can simply the operation process effectively.

The host material made of HCNs@TiN exhibits several advantages as follows: first, the large inner cavity structure of hollow carbon nanospheres not only allows a high loading of sulfur but also effectively buffer the large volume change of sulfur during the procedure of lithiation and delithiation process. Second, the outer carbon shell can provide an effective conductive network to improve the electronic conductivity of sulfur cathodes and serves as a physical barrier to restrain the dissolution of polysulfides. Third, the well-distributed TiN particles coated on HCNs can act as an anchor to confine the shuttling effect of LiPSs via strong chemical interactions. As a consequence of these 
favorable effects, the developed HCN@TiN-S composite with 62.86 wt\% sulfur content delivers a high initial discharge specific capacity of $1097.8 \mathrm{mAh} \mathrm{g}^{-1}$ at $0.1 \mathrm{C}$ with coulombic efficiency $(\approx 98 \%)$, and retains a discharge capacity of $812 \mathrm{mAh} \mathrm{g}^{-1}$ after 200 cycles, which are much better than those of the contrast HCNs-S electrodes.

\section{Experimental Section}

\subsection{Preparation of PACP hollow nanospheres}

The PACP hollow spheres were prepared by an oxidative polymerized method reported in previous works [31-33]. In particular, the polymerization was carried out in a thermal incubator at $0{ }^{\circ} \mathrm{C}$. Initially, $0.9 \mathrm{~g}$ Triton X-100 and $900 \mathrm{ml}$ deionized water were added to a beaker covered with polyethylene film and stirred 20 minutes. Then, $4.35 \mathrm{~mL}$ pyrrole and $5.7 \mathrm{~mL}$ aniline were added to the mixed solution. After 10 minutes, $120 \mathrm{~mL}$ of aqueous solution containing $27.45 \mathrm{~g}$ of ammonium persulphate as an initiator was added forming a homogeneous solution. After the reaction lasted for $12 \mathrm{~h}$, the resulting precipitate was vacuum filtered, washed with deionized water several times and dried at $80{ }^{\circ} \mathrm{C}$ for $6 \mathrm{~h}$. Finally, the sample was collected for further processing.

\subsection{Synthesis of PACP@ $\mathrm{TiO}_{2}$}

$\mathrm{TiO}_{2}$ layer was coated on PACP copolymer hollow nanospheres via a sol-gel method. Typically, $0.5 \mathrm{~g}$ of PACP was dispersed in a serum bottle with $150 \mathrm{~mL}$ of ethanol solution through sonication. After stirring for $30 \mathrm{~min}, 360 \mu \mathrm{L}$ of tetrabutyl titanate (TBOT) in ethanol (the total solution volume is $50 \mathrm{~mL}$ ) was added drop by drop under vigorous stirring and sealed immediately. After $1 \mathrm{~h}$, twisting the lid off, making the mixed solution in full contact with the air, the hydrolysis reaction was carried out at room temperature for $12 \mathrm{~h}$ under magnetic stirring. Finally, the PACP@ $\mathrm{TiO}_{2}$ hollow nanospheres could be obtained by vacuum filtration, washing with deionized water three 
times and drying at $80^{\circ} \mathrm{C}$ for $12 \mathrm{~h}$.

\subsection{Synthesis of HCNs@ TiN by microwave}

At first, 0.5g PACP@ $\mathrm{TiO}_{2}$ was placed within a graphite crucible and inserted into a silicon carbide $(\mathrm{SiC})$ tube which has a critical auxiliary heating effect. Then, the $\mathrm{SiC}$ tube was wrapped with asbestos and placed in a microwave furnace. The furnace was heated to $1200{ }^{\circ} \mathrm{C}$ at a very high rate of heating (generally within 5 min) under a $\mathrm{N}_{2}$ atmosphere with a gas pressure of $20 \mathrm{kPa}$. Then the target temperature was maintained for 20 minutes and then furnace cooling. For comparison, the control group HCNs was prepared by direct carbonization of PACP under the same heating condition.

\subsection{Characterization}

Scanning electron microscopy (SEM) was carried out on a field emission SU-70 microscope. TEM images were obtained by a JEOL JEM2010 electron microscope. Wide-angle X-ray diffraction (XRD) patterns were acquired with a Bruker D8 Advance diffractometer using monochromated $\mathrm{Cu}-\mathrm{K}$ radiation $(40 \mathrm{kV}, 200 \mathrm{~mA})$. Data was collected from $2 \theta=20^{\circ}$ to $80^{\circ}$ in a step of 0.02 with a scanning rate of $0.2^{\circ} \mathrm{s}^{-1}$. Thermogravimetric (TG) analysis was performed on TGA-Q50 thermal gravimetric analyzer in a $\mathrm{N}_{2}$ atmosphere at heating rate $10{ }^{\circ} \mathrm{C} \mathrm{min}^{-1}$. Raman spectra were collected using a Renidhaw inia Raman Microscope with an excitation laser wavelength of $\lambda=$ $514.5 \mathrm{~nm}$. The $\mathrm{N}_{2}$ adsorption/desorption isotherms were measured using an ASAP 2020 (Micromeritics Co.) at $77 \mathrm{~K}$. The surface area was calculated according to the BrunauerEmmett-Teller (BET) model based on adsorption data in the partial pressure (P/Po) range of $0.10-0.20$. The total pore volumes and pore-size distributions were estimated from the amount of nitrogen adsorbed at $\mathrm{P} / \mathrm{Po}=0.99$. The pore size distribution was calculated by the original density functional theory (DFT). X-ray photoelectron spectroscopy (XPS) was conducted on a Microlab 350 spectrometer employing a 
monochromatic Mg-Ka X-ray source.

\section{5 cell fabrication and electrochemical performance test}

For the electrochemical measurement of these composites, the HCNs@TiN and HCNs were admixed with acetylene black and LA133 binder in a weight ratio of $80: 15: 5$ to form a homogeneous slurry and then the slurry was blade cast onto aluminum foils and dried at $60{ }^{\circ} \mathrm{C}$ for $12 \mathrm{~h}$ under vacuum and then cut into discs with a typical diameter of $16 \mathrm{~mm}$. The mass loading of sulfur in these working electrodes were approximately $1 \mathrm{mg} \mathrm{cm}^{-2}$. The 2016 coin cells assembly was conducted in an Ar-filled glove box $\left(\mathrm{O}_{2}\right.$ and $\mathrm{H}_{2} \mathrm{O}$ level $\left.<0.1 \mathrm{ppm}\right)$. The separator was Celgard2400 (America) micro-porous polypropylene membrane and Li metal was used as the counter electrodes. The electrolyte was composed of $1 \mathrm{M}$ bis(trifluoromethane) sulphonamide lithium salt and $0.1 \mathrm{M} \mathrm{LiNO}_{3}$ in a mixture of 1,3-dioxolane and 1,2- dimethoxyethane (1:1 by volume). Galvanostatic discharge-charge tests were conducted on a Land automatic batteries tester (Land CT2001A, Wuhan, China) with voltage window of 1.8-3.0 V versus $\mathrm{Li}^{+} / \mathrm{Li}$. The current density was based on the weight of sulfur $\left(1 \mathrm{C}=1670 \mathrm{~mA} \mathrm{~g}^{-}\right.$ $\left.{ }^{1}\right)$. Cyclic voltammetry (CV) and electrochemical impedance spectroscopy (EIS) was performed on an electrochemistry workstation (CHI660D).

\section{Results and discussion}

Fig.1 outlines the procedure for the preparation of HCNs@TiN. Initailly, the PACP hollow nanospheres were synthesized through a one-step route by the polymerization of pyrrole and aniline in Triton X-100 solution. As shown in the SEM images in Fig. 2a, the PACP nanospheres with uniform size and smooth surface are obtained. The TEM image (Fig. 2b) of PACP shows hollow nanospheres with an average inner diameter of $60 \mathrm{~nm}$ and an average outer diameter of $120 \mathrm{~nm}$. Fig. 2c exhibits that PACP is amorphous structure and no lattice fringe of graphite has been observed. 


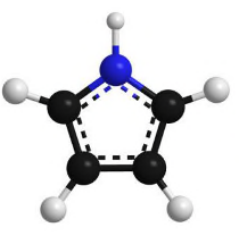

Pyrrole

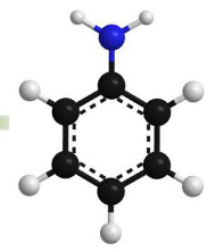

Aniline
Tritonx-100

Ammonium persulfate

- $\mathrm{C} \bullet \mathrm{N}$

- $\mathbf{H}$

- PACP

- $\mathrm{TiO}_{2}$

- TiN

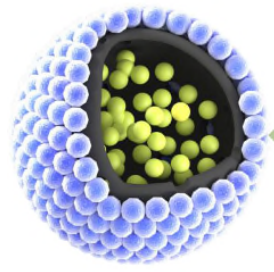

C@TiN

\section{Sulfur \\ Loading}

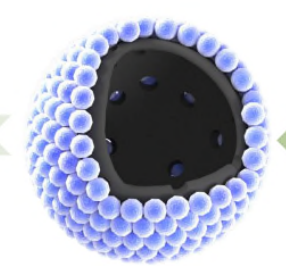

C@TiN

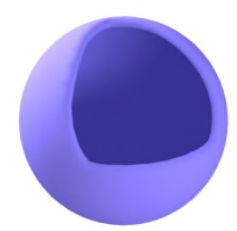

PACP

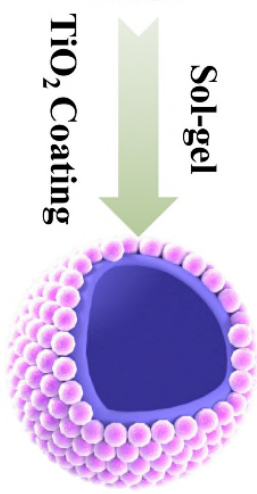

PACP@TiO

Fig.1 A schematic illustration of the procedure for the preparation of HCNs@TiN-S.

After that, selecting tetrabutyl titanate as a titanium source, a thin uniform layer of amorphous $\mathrm{TiO}_{2}$ was coated on the surface of PACP hollow nonospheres via a sol-gel reaction. Then the obtained $\mathrm{PACP} @ \mathrm{TiO}_{2}$ composite was quickly heated to the $1200{ }^{\circ} \mathrm{C}$ and treated for 10 min through microwave heating reduction under a $\mathrm{N}_{2}$ atmosphere, and HCN@TiN particles were obtained.Fig.2(d and e) show that HCNs@TiN retained a perfect nanospherical morphology but presented a smaller average inner diameter of about $50 \mathrm{~nm}$ and an average outer diameter of about $100 \mathrm{~nm}$. It indicated that a large number of oxygen-containing functional groups of PACP are lost in the process of microwave reduction. In contrast to the smooth surface of PACP, many nanoparticles which had a size distribution of 10-50 nm were homogeneously decorated on the surface of the hollow carbon sphere. The control group HCNs had a smooth surface and presented an average inner diameter of about $50 \mathrm{~nm}$ and an average outer diameter of about 96 nm, as shown in Fig. S1. HRTEM images obtained from three regions (I, II, III in fig. 2f) clearly show that some lattice fringes appear on the carbon matrix and 
nanoparticles. The lattice fringe in region "I" was found to be ca. $0.335 \mathrm{~nm}$, corresponding to the $\mathrm{d}_{002}$ spacing of graphite. Obviously, graphite crystallites was generally observed in the carbon spherical shell after microwave reduction (region I), which helps to enhance the electrical conductivity of carbon matrix. In addition, the lattice fringes in the region "II" and "III" were measured to be ca. 0.213 and $0.245 \mathrm{~nm}$, corresponding to $\mathrm{d}_{200}$ and $\mathrm{d}_{111}$ spacing of TiN, respectively, which are good in agreement with the result by XRD (Fig. 4). Finally, HCNs@TiN-S composites were synthesized by a melt-diffusion method that encapsulates the sublimed sulfur into HCNs@TiN. The SEM images of HCNs-S and HCNs@TiN-S are shown in Fig.3. The corresponding elemental maps of HCNs@TiN-S indicate the homogeneous distribution of sulfur in this composite.
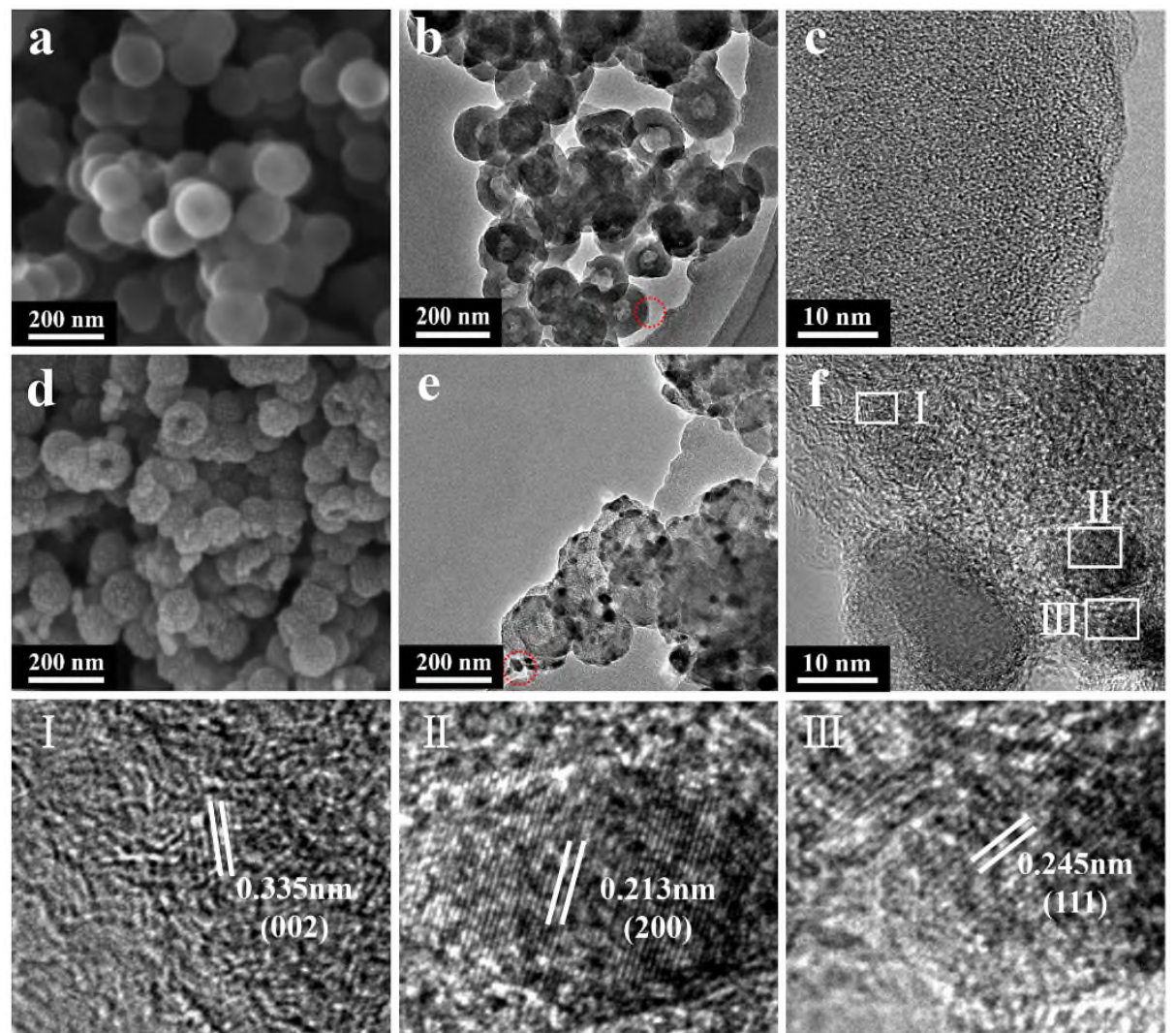

Fig.2 (a) SEM image and (b, c) TEM images of PACP; (d) SEM image and (e, f) TEM images of HCNs@TiN; (I-III) the corresponding HRTEM images marked with white rectangle in figure f.

The crystal structure of the samples was analyzed by X-ray diffraction (XRD). As 
shown in Fig. 4, the HCNs samples exhibit two broad peaks that can be observed at $2 \theta$ values of at around $25^{\circ}$ and $43^{\circ}$ with no detectable crystalline Bragg peaks, which is characteristic for amorphous structure. While five well-defined characteristic peaks at $36.5^{\circ}, 42.4^{\circ}, 61.5^{\circ}, 73.7^{\circ}$ and $77.6^{\circ}$ for the (111), (200), (220), (311) and (222) planes corresponding to crystalline TiN (JCPDS no. 65-0414) were detected for the HCNs@TiN samples. The sharp bragg peaks indicate that the as-prepared TiN nanoparticles have good crystallinity. The peaks of elemental sulfur (JCPDS no. 741465) are visible in these two composites HCNs-S and HCNs@TiN-S. However, there is a peak at $20^{\circ}$ for these tow samples, which match neither sulfur nor TiN. This peak may be ascribed to a different form of sulfur, namely rosickyit-type sulfur (JCPDS no. 13-0141), suggesting that S8 is not the only form of sulfur present after sulfur infiltration by the melt-diffusion method [35].

The reaction involving transformation of $\mathrm{TiO}_{2}$ into $\mathrm{TiN}$ nanoparticles could be described by the following equations:

$$
2 \mathrm{TiO}_{2}+4 \mathrm{C}+\mathrm{N}_{2} \rightarrow 2 \mathrm{TiN}+4 \mathrm{CO} \uparrow
$$

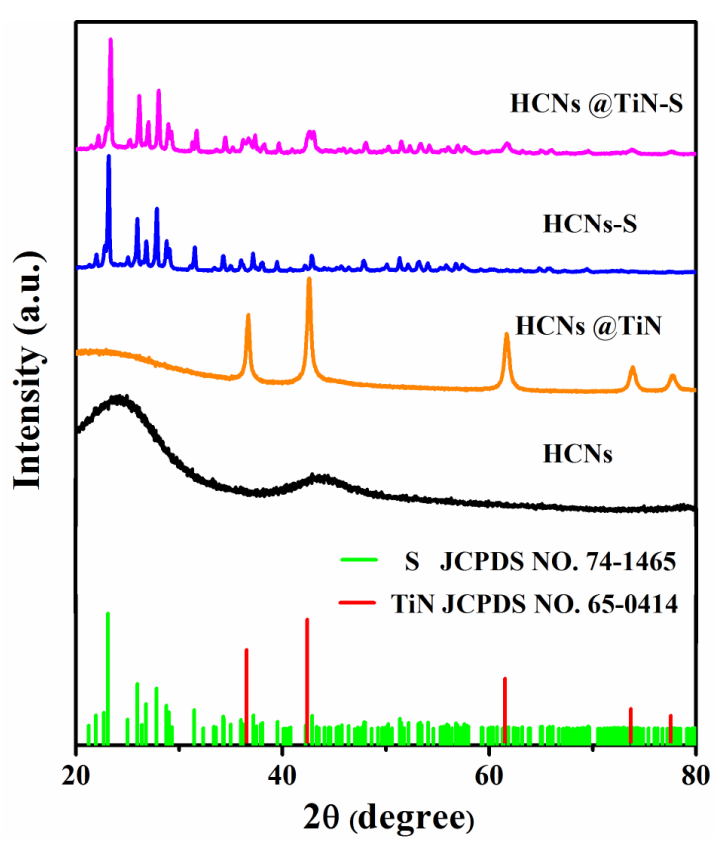


Fig.4 XRD pattern of HCNs, HCNs@TiN, HCNs-S and HCNs@TiN-S. Green and red drop lines correspond to Sulfur (JCPDS no. 74-1465) and TiN (JCPDS no. 65-0414)

It is known that approximately $78 \%$ by volume of air is $\mathrm{N}_{2}$. Due to the low cost and high safety, we choose N4 instead of NH3 to provide a nitrogen source for the nitridation of $\mathrm{TiO} 2$. Since $\mathrm{N} 2$ has a quite high chemical stability, it is very difficult to quickly obtain $\mathrm{TiN}$ below $1300{ }^{\circ} \mathrm{C}$ by conventional carbothermal reduction of $\mathrm{TiO} 2$ under an N2 atmosphere. In this work, however, only 10 min was taken to synthesize HCN@TiN particles with high purity at $1200{ }^{\circ} \mathrm{C}$ by the microwaves heating process. It is considered that the uniform and rapid inside out heating profile leads to a shortened reaction time. On the other hand, the accelerated diffusion between reacants by the nonthermal effects of microwaves has contributed to lower initial reaction temperature. Additionally, if the N2 atmosphere is replaced by argon gas (Ar), the product of the reaction transforms into HCNs@:TiC (Fig. S2). The results indicate that the $\mathrm{N}$ in the as-prepard TiN particles comes form N2 rather than PACP itself.

By performing the thermogravimetric analysis (TGA) from room temperature to $450^{\circ} \mathrm{C}$ at a heating rate of $10^{\circ} \mathrm{C} \min ^{-1}$ under a $\mathrm{N}_{2}$ atmosphere (Fig. 4a). The sulfur contents in the HCNs-S and HCNs@TiN-S composite were determined to be $61.68 \%$ wt $\%$ and $62.86 \mathrm{wt} \%$, respectively.
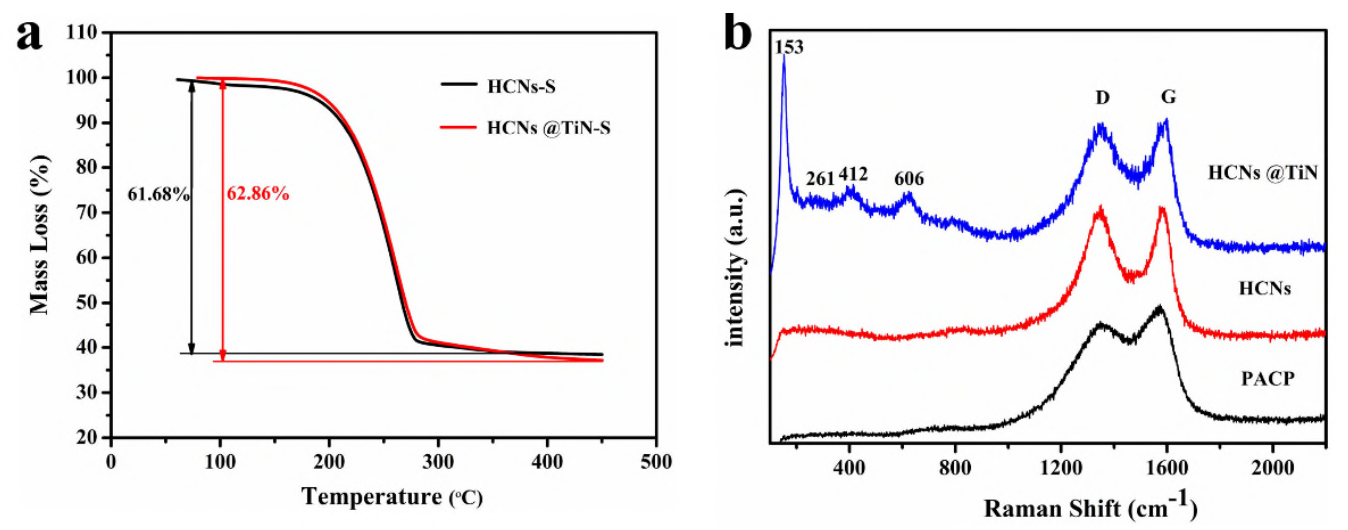

Fig.4 (a) TG curves of HCNs-S and HCNs@TiN-S composite; (b) Raman spectra of PACP, HCNs and HCNs@TiN. 
Raman spectra are depicted in Fig. 5b. Four peaks around 153, 261, 412, $606 \mathrm{~cm}^{-1}$ correspond to the bending and stretching modes of TiN, which are consistent with the results in the previous report [36]. Moreover,, two characteristic peaks located at about 1350 and $1587 \mathrm{~cm}^{-1}$ were observed in the synthesized PACP, HCNs and HCNs@TiN, which respectively correspond to the D-band (disorder and defects) and G-band (graphitic) of carbon. The D-band usually corresponds to the vibrations of $\mathrm{sp}^{3}$ hybridized carbon, while the G-band is related to vibrations of $\mathrm{sp}^{2}$-bonded ordered graphitic carbon atoms. The distanct G bands existing in HCNs and HCNs@TiN indicate that these materials may have good electrical conductivity, which will be favorable for the fast transmission of electrons during the electrochemical reaction process [37]. The nitrogen $\left(\mathrm{N}_{2}\right)$ adsorption-desorption isotherms and pore size distribution profiles of PACP, HCNs and HCNs@TiN are shown in Fig. S3. The BET surface and total volume of PACP were calculated to be $39.4 \mathrm{~m}^{2} \mathrm{~g}^{-1}$ and $0.16 \mathrm{~cm}^{3} \mathrm{~g}^{-1}$, respectively, which increased to $54.9 \mathrm{~m}^{2} \mathrm{~g}^{-1}$ and $0.204 \mathrm{~cm}^{3} \mathrm{~g}^{-1}$ when transformed into HCNs@TiN (Table S1).

XPS measurements were carried out to confirm the surface compositions of HCNs@TiN. The survey spectra and high resolution Ti2p XPS spectra are shown in Fig.5 (a-b). After deconvolution of the Ti2p spectra, six peaks referring to three pairs of spin-orbit split doublets are detected from the HCNs@TiN, including Ti-N bond (Ti $2 \mathrm{p}_{3 / 2}, 455.6 \mathrm{eV}$; Ti $2 \mathrm{p}_{1 / 2}, 461.3 \mathrm{eV}$ ), Ti-O bond (Ti $2 \mathrm{p}_{3 / 2}, 458.5 \mathrm{eV}$; Ti $2 \mathrm{p}_{1 / 2}, 464.2 \mathrm{eV}$ ), Ti-N-O bond (Ti 2 $\mathrm{p}_{3 / 2}, 456.9 \mathrm{eV}$; Ti 2 $\mathrm{p}_{1 / 2}, 462.6 \mathrm{eV}$ ) [38, 39]. Based on these data, oxide/oxynitride passivation layer was formed on the surface of TiN when the sample is exposed to ambient air. Previous research has shown that the hydrophilic Ti-O groups can provide a polar surface that would form strong chemical bonds with the polysulfde [40, 43, 39]. Moreover, the N 1s detailed spectrum of HCNs@TiN is shown in Fig.5 c, 
presenting a broad peak can be split into two peaks at 397.0 and $397.4 \mathrm{eV}$ associated with Ti-N and Ti-N-O bond, respectively, which is consistent with the Ti $2 p$ spectrum [39]. Another small peak at higher binding energy can be ascribed to the chemisorbed nitrogen.
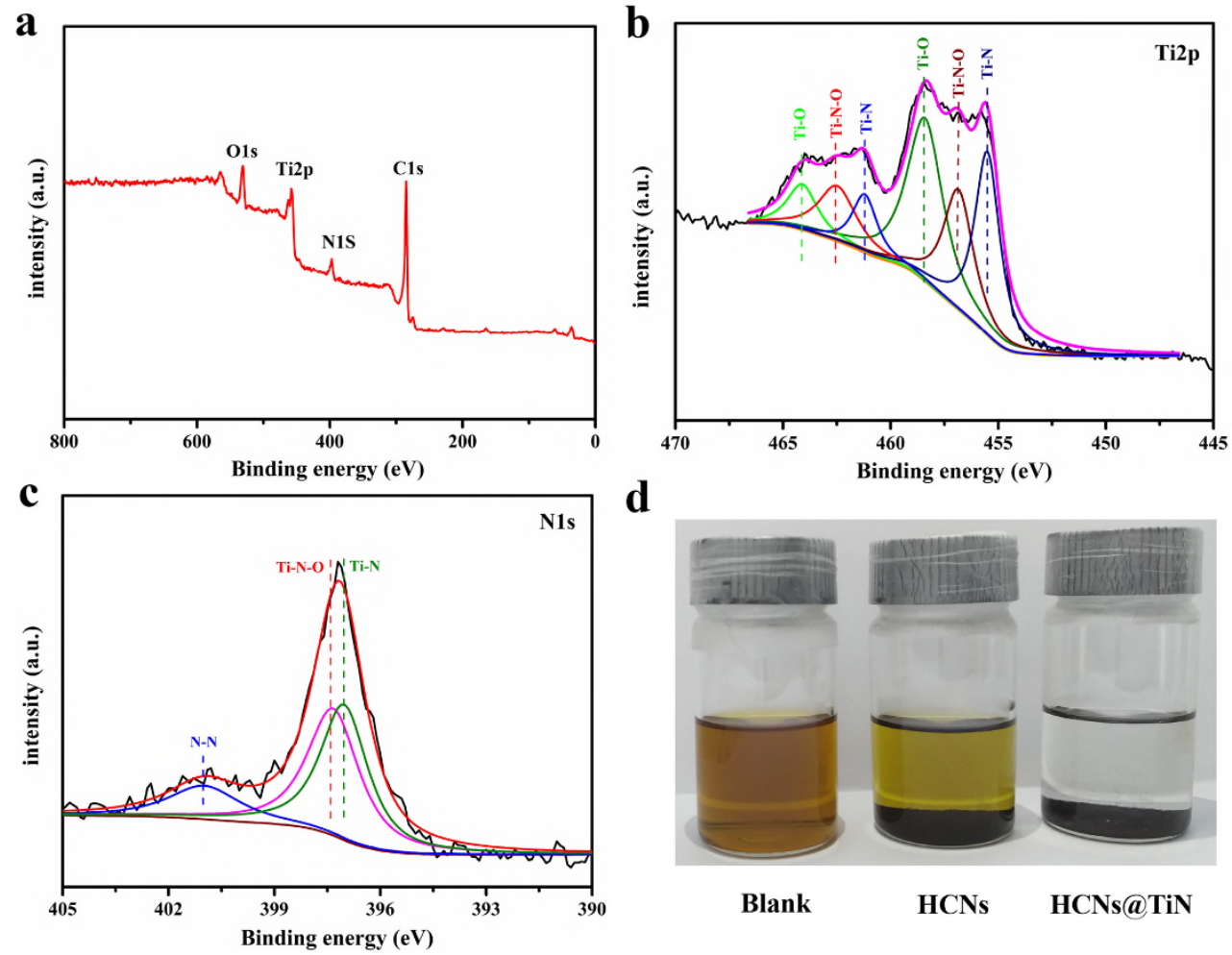

Fig.5 (a-c) The XPS spectra of HCNs@TiN, (a)XPS survey spectra of HCNs@TiN, (b) Ti2p XPS spectra of HCNs@TiN , (c) N1s XPS spectra of HCNs@TiN ; (d) Digital picture of the Li $2 \mathrm{~S}_{6}$ static adsorption by HCNs and HCNs@TiN in DOL/DME (1:1, v/v) solution for 36h.

The surface interaction between HCNs@TiN and Li2Sx was proved through visualized adsorption experiments. Typically, 20 mg HCNs and HCNs@TiN were added into a one sealed vial containing a $10 \mathrm{ml}$ solution of $\mathrm{Li}_{2} \mathrm{~S}_{6}(10 \mathrm{mmol})$ in $1,3-$ dioxolane (DOL) and 1,2-dimethoxyethane (DME), and the DOL/DEM is 1: 1 by volume. As shown in Fig. 6d, after standing for $36 \mathrm{~h}$, the yellow Li2S6 solution containing HCNs@TiN becomes colourless, confirming that the HCN@TiN host can effectively trapthe Li2Sx during the charge/discharge process. However, comparing the solutions for the control groups, there is no colour change for the blank one, while for 
the HCN sample, the color of the Li2S6 solution only changes from yellow to pale yellow. The experiment demonstrates that the polar TiN has a stronger adsorption ability for LiPSs than carbon materials.

To compare the electrochemical performance, HCNs@TiN-S and HCNs-S composites were respectively made as cathodes and Li metal as the anode in 2016 coin cells. Fig. 7a shows the corresponding profiles for the 1 st, 2 nd, and 3 rd cycles in the voltage window of 1.8-3.0 V with a scan rate of $0.2 \mathrm{mVs}^{-1}$. During the cathodic scan in the CV profiles, two well-defined characteristic reduction peaks are located at $2.27 \mathrm{~V}$ and $2.03 \mathrm{~V}$, which correspond to the transformation of the open ring of $\mathrm{S}_{8}$ molecules to form long chain Li2PSs $\left(\mathrm{Li}_{2} \mathrm{~S}_{\mathrm{n}}, 4 \leq \mathrm{n}<8\right)$, and then to the insoluble $\mathrm{Li}_{2} \mathrm{~S}_{\mathrm{n}}(\mathrm{n}=1$ or 2$)$, respectively. In reverse anodic scan process, two oxidization peaks at $2.34 \mathrm{~V}$ and $2.39 \mathrm{~V}$ were related to reversible conversion of solid $\mathrm{Li}_{2} \mathrm{~S}$ or $\mathrm{Li}_{2} \mathrm{~S}_{2}$ firstly oxidized to shortchain $\mathrm{Li}_{2} \mathrm{~S}_{\mathrm{n}}(\mathrm{n}=3$ or 4$)$, and then further oxidized to long chain $\mathrm{Li}_{2} \mathrm{~S}_{8}$ or cyclo-S $\mathrm{S}_{8}$. After one cycle, the CV peak positions of the second and third cycles almost overlapped, implying the outstanding electrochemical reversibility of HCNs@TiN-S. 

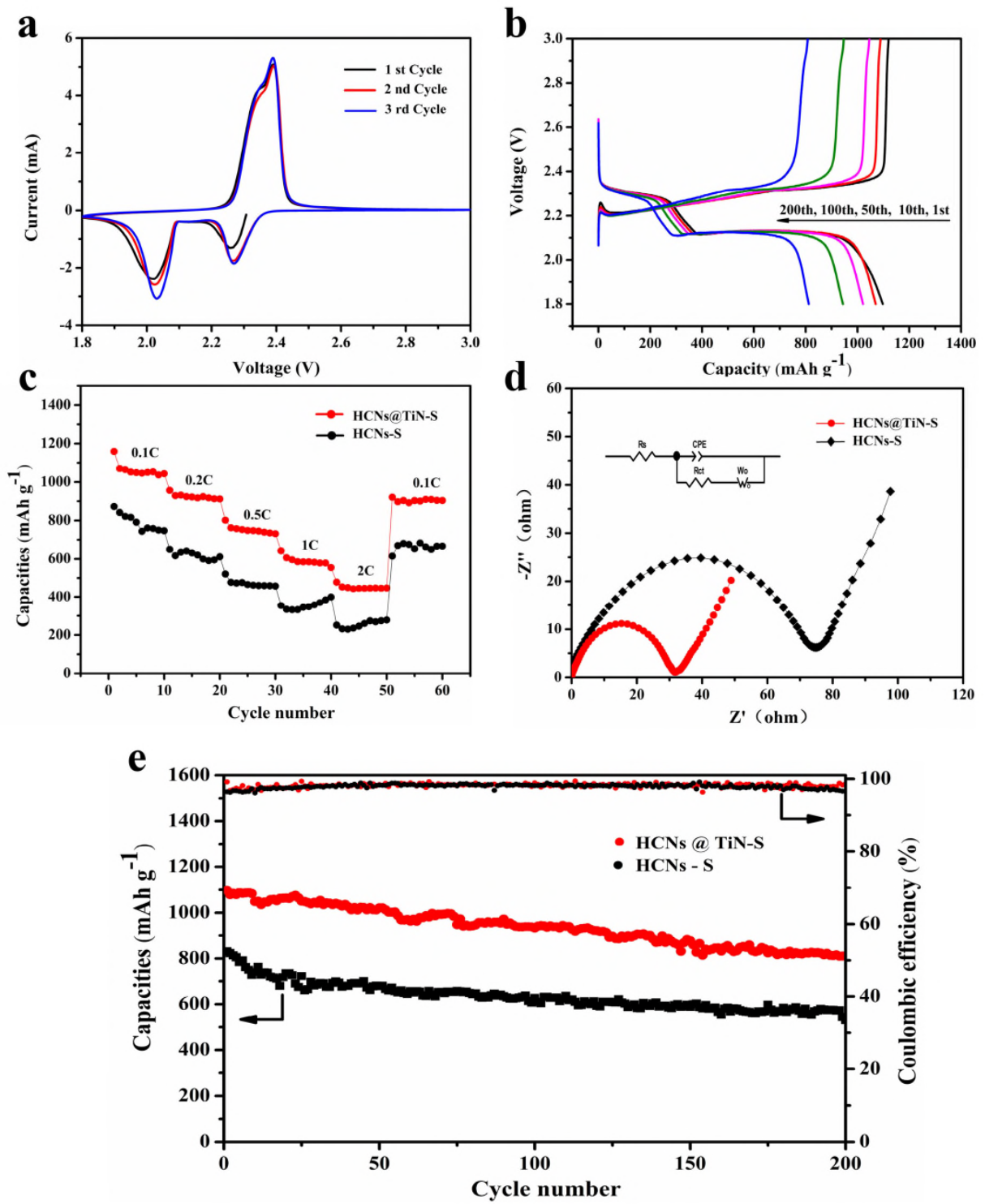

Fig.6 (a) Typical CV profiles and (b) charge-discharge profiles of HCNs@TiN-S cathode over different cycles at a current rate of 0.1C; (c) rate capacity of HCNs-S and HCNs@TiN-S, (d) EIS spectra before cycling and (e) cycling performance of HCNs-S and HCNs@TiN-S cathode at 0.1C.

Fig. 7b shows the galvanostatic charge/discharge profiles of the HCNs@TiN-S composite electrode over different cycles at a rate of $0.1 \mathrm{C}$. The profiles show two discharge plateaus at around 2.3 and 2.1 V, representing the different stages of the redox reactions of sulfur. These results are basically in accordance with the $\mathrm{CV}$ results discussed above.

The rate capacities of the two composite electrodes were evaluated at different 
discharge rates from 0.1 to 2C, as shown in Fig. 7c. It can be seen that the HCNs@TiN$\mathrm{S}$ cathode shows much better rate capacity than that of the HCNs-S cathode. The average discharge capacity of HCNs@TiN-S at 0.1, 0.2, 0.5, 1 and 2 C are 1062, 925, 752, 589 and $450 \mathrm{mAh} \mathrm{g}^{-1}$, respectively. Then, when the current rate switches back to $0.1 \mathrm{C}$, the specific capacity can be largely recovered to $921 \mathrm{mAh} \mathrm{g}^{-1}$ after 50 cycles. In contrast, the HCNs-S electrode exhibits obviously lower discharge capacities, the specific capacity at $0.1 \mathrm{C}, 0.2 \mathrm{C}, 0.5 \mathrm{C}, 1 \mathrm{C}, 2 \mathrm{C}$ rates are $790,619,471,355$ and $255 \mathrm{mAh}$ $\mathrm{g}^{-1}$, respectively.

To further understand the different kinetics of the HCNs@TiN-S and HCNs-S cathode during the electrochemical reaction, EIS data of both electrodes are collected before cycling in a frequency range of $10 \mathrm{mHz}$ to $100 \mathrm{KHz}$. As shown in Fig. 6d, it can be observed that both composite electrodes are composed of a semicircle in the high frequency region representing the charge transfer process, and a sloped line in the low frequency region corresponding to a semi-infinite Warburg diffusion process, which is related to the ion diffusion in the electrode. The charge transfer resistance $(R c t)$ of the HCNs@TiN-S (17.92 $\Omega$ ) is lower than that of HCNs-S (77.03 $\Omega$ ). Because of both cathodes containing approximately the same content of sulfur, the different charge transfer resistance could be attributed to the well-distributed coating TiN particles on HCNs, which shows better ability to facilitate the charge transfer for surface reaction than the single carbon materials.

The long-term cycling performance of the HCNs@TiN-S and HCNs-S cathode materials was measured at a rate of $0.1 \mathrm{C}$. As shown in Fig. 7e, the HCNs-S electrode suffers from a sever capacity fade with a low capacity of $529.9 \mathrm{mAh} \mathrm{g}^{-1}$ after 200 cycles, showing a high decay rate of $0.18 \%$ and a low capacity retention of $63.7 \%$ of the original capacity $\left(831.3 \mathrm{mAh} \mathrm{g}^{-1}\right)$. In contrast, the original specific discharge capacity 
of the HCNs@TiN-S electrode is 1097.8 mAh g $^{-1}$ and remains stable in the initial cycles. After 200 cycles, a reversible capacity of $812.6 \mathrm{mAh} \mathrm{g}^{-1}$ is still retained, corresponding to $74 \%$ capacity retention of the original capacity and $0.13 \%$ decay rate per cycle, proving its relatively high specific capacitance and good long-term cycling stability. Better cycling performance of the HCNs@TiN-S electrode further confirms that the additional strong polar chemical interaction of TiN nanoparticles instead of only weak physical entrapment of carbon materials could effectively mitigate the shuttling effect of the polar LiPSs. Furthermore, compared with some similar sulfur cathodes that are summarized in Table S2, [15, 32, 44-46] the HCN@TiN-S electrode in this work also exhibits an attractive cycling stability.

\section{Conclusions}

In summary, to enhance the interaction with LiPSs and electrochemical performance of the cathode materials for Li-S batteries, we present an efficient strategy to bind TiN nano-particles to hollow carbon nanospheres by the sol-gel and microwave reduction nitridation methods. Through this way, we can quickly obtain well-crystallized TiN nano particles at a lower cost. Benefitting from the unique structural and outstanding functional behavior of the components, the as-prepared HCNs@TiN-S cathode material with $62.86 \mathrm{wt} \%$ sulfur content exhibits a high specific capacity of $1097.8 \mathrm{mAh} \mathrm{g}^{-1}$ at $0.1 \mathrm{C}$ with excellent coulombic efficiency $(\approx 98 \%)$, and retain a discharge capacity of $812.6 \mathrm{mAh} \mathrm{g}^{-1}$ afer 200 cycles at 0.1C. Testing results shows that, the HCNs@TiN composite can effectively alleviate the shuttle effects of polysulfide and enhance the utilization of sulfur compared with the HCNs without modification. It can be expected that other highly conductive metal nitrides could also act as electrode additives in Li-S batteries as shown in TiN in this work.

\section{Conflicts of interest}


There are no conflicts to declare.

\section{Acknowledgments}

This work was financially supported by the National Natural Science Foundation of

China (No. 51202150 and 51272161), foundation of the State Key Laboratory of Solidification Processing in NWPU (SKLSP201110) and Shenzhen Basic Research Program (No. JCYJ2016042209 1418366).

\section{References}

[1] A. Manthiram, Y. Fu, S.H. Chung, C. Zu, Y.S. Su, Chemical Reviews, 114 (2014) 11751-11787.

[2] D. Bresser, S. Passerini, B. Scrosati, Chemical Communications, 49 (2013) 10545-10562.

[3] Y.X. Yin, S. Xin, Y.G. Guo, L.J. Wan, Angewandte Chemie, 52 (2013) 13186-13200.

[4] Z. Li, Y. Huang, L. Yuan, Z. Hao, Y. Huang, Carbon, 92 (2015) 41-63.

[5] S. Rehman, S. Guo, Y. Hou, Advanced Materials, 28 (2016) 3167-3172.

[6] X. Liang, Z. Wen, Y. Liu, H. Zhang, L. Huang, J. Jin, Journal of Power Sources, 196 (2011) 3655-3658.

[7] T.P. Mcnicholas, L. Ding, D. Yuan, J. Liu, Nano Letters, 9 (2009) 3646-3650.

[8] R. Elazari, G. Salitra, A. Garsuch, A. Panchenko, D. Aurbach, Advanced Materials, 23 (2011) 56415644.

[9] S.Z. Zeng, X. Zeng, W. Tu, Y. Yao, L. Yu, H. Wu, W. Jin, H. Huang, J. Zou, Journal of Materials Chemistry A, 5 (2017).

[10] R. Chen, T. Zhao, J. Lu, F. Wu, L. Li, J. Chen, G. Tan, Y. Ye, K. Amine, Nano Letters, 13 (2013) $4642-$ 4649 .

[11] G. He, S. Evers, X. Liang, M. Cuisinier, A. Garsuch, L.F. Nazar, Acs Nano, 7 (2013) 10920-10930.

[12] F. Pei, T. An, J. Zang, X. Zhao, X. Fang, M. Zheng, Q. Dong, N. Zheng, Advanced Energy Materials, 6 (2016) n/a-n/a.

[13] N. Jayaprakash, J. Shen, S.S. Moganty, A. Corona, L.A. Archer, Angewandte Chemie, 50 (2011) 59045908.

[14] W.S. Zhi, H. Wang, P.C. Hsu, Q. Zhang, W. Li, G. Zheng, H. Yao, Y. Cui, Energy \& Environmental Science, 7 (2014) 672-676.

[15] S. Rehman, S. Guo, Y. Hou, Advanced Materials, 28 (2016) 3167-3172.

[16] Y. Zhang, Y. Zhao, A. Yermukhambetova, Z. Bakenov, P. Chen, Journal of Materials Chemistry A, 1 (2013) 295-301.

[17] H. Wang, Q. Zhang, H. Yao, Z. Liang, H.W. Lee, P.C. Hsu, G. Zheng, Y. Cui, Nano Letters, 14 (2014) 7138-7144.

[18] S.S. Zhang, D.T. Tran, Journal of Materials Chemistry A, 4 (2016) 4371-4374.

[19] J. Zhang, H. Hu, Z. Li, X.W. Lou, Angewandte Chemie International Edition, 55 (2016) 3982-3986.

[20] Q. Li, Z. Li, Z. Zhang, C. Li, J. Ma, C. Wang, X. Ge, S. Dong, L. Yin, Advanced Energy Materials, 6 (2016) 1600376.

[21] S.H. Al, G. Babu, C.V. Rao, L.M. Arava, Journal of the American Chemical Society, 137 (2015) 11542. 
[22] X. Liang, A. Garsuch, L.F. Nazar, Angewandte Chemie, 127 (2015) 3979-3983.

[23] Z. Sun, J. Zhang, L. Yin, G. Hu, R. Fang, H.M. Cheng, F. Li, Nature Communications, 8 (2017) 14627.

[24] F. Zhou, Z. Li, X. Luo, T. Wu, B. Jiang, L.L. Lu, H.B. Yao, M. Antonietti, S.H. Yu, Nano Letters, 18 (2018).

[25] Z. Cui, C. Zu, W. Zhou, A. Manthiram, J.B. Goodenough, Advanced Materials, 28 (2016) 6926-6931.

[26] Y. Wang, R. Zhang, Y.C. Pang, X. Chen, J. Lang, J. Xu, C. Xiao, H. Li, K. Xi, S. Ding, Energy Storage Materials, (2018).

[27] X. He, Y. Shuai, L. Na, K. Chen, Y. Zhang, Z. Zhang, F. Gan, Materials Letters, 215 (2018) 91-94.

[28] P. Sun, R. Lin, Z. Wang, M. Qiu, Z. Chai, B. Zhang, H. Meng, S. Tan, C. Zhao, W. Mai, Nano Energy, 31 (2016).

[29] P. Qin, X. Li, B. Gao, J. Fu, L. Xia, X. Zhang, K. Huo, W. Shen, P.K. Chu, Nanoscale, 10 (2018).

[30] D.R. Deng, T.H. An, Y.J. Li, Q.H. Wu, M.S. Zheng, Q.F. Dong, Journal of Materials Chemistry A, 4 (2016).

[31] S.Z. Zeng, Y. Yao, X. Zeng, Q. He, X. Zheng, S. Chen, W. Tu, J. Zou, Journal of Power Sources, 357 (2017).

[32] F. Xu, Z. Tang, S. Huang, L. Chen, Y. Liang, W. Mai, H. Zhong, R. Fu, D. Wu, Nature Communications, 6 (2015) 7221.

[33] C. Zhou, J. Han, G. Song, R. Guo, Journal of Polymer Science Part A Polymer Chemistry, 46 (2010) 3563-3572.

[34] C.E. Bamberger, G.M. Begun, Applied Spectroscopy, 43:1 (1989) 134-138.

[35] M. Fang, Z. Chen, Y. Liu, J. Quan, C. Yang, L. Zhu, Q. Xu, Q. Xu, Journal of Materials Chemistry A, 6 (2017).

[36] T. Lan, J. Dou, F. Xie, P. Xiong, M. Wei, Journal of Materials Chemistry A, 3 (2015) 10038-10044.

[37] K.S. Robinson, P.M.A. Sherwood, Surface \& Interface Analysis, 6 (2010) 261-266.

[38] M.H. Chan, F.H. Lu, Thin Solid Films, 517 (2009) 5006-5009.

[39] Y. Sun, Y. Zhao, Y. Cui, J. Zhang, G. Zhang, W. Luo, W. Zheng, Electrochimica Acta, (2017).

[40] X. Song, T. Gao, S. Wang, Y. Bao, G. Chen, L.X. Ding, H. Wang, Journal of Power Sources, 356 (2017) 172-180.

[41] L. Z, Z. J, G. B, W. D, L. LM, L. XW, Nature Communications, 7 (2016) 13065.

[42] W.S. Zhi, W. Li, J.J. Cha, G. Zheng, Y. Yuan, M.T. Mcdowell, P.C. Hsu, C. Yi, Nature Communications, 4 (2013) 1331.

[43] K.S. Robinson, N.u.T. Univ., University of Newcastle Upon Tyne, (1986). 


\title{
Supporting Information
}

\section{Microwave-assisted rapid preparation of TiN nanoparticles coated hollow carbon nanospheres for lithium-sulfur batteries}

\author{
Jianxin $\mathrm{Tu}^{\mathrm{a}, \mathrm{b}}$, HeJun Li ${ }^{\mathrm{a}}$, JiZhao Zou ${ }^{\mathrm{b} *}$, ShaoZhong Zeng ${ }^{\mathrm{b}}$, \\ Qi Zhang ${ }^{\mathrm{c}}$, Liang $\mathrm{Yu}^{\mathrm{b}}$ and Xierong Zeng ${ }^{\mathrm{a}, \mathrm{b}^{*}}$
}

${ }^{\text {a }}$ State Key Laboratory of Solidification Processing, Carbon/Carbon Composites

Research Center, Northwestern Polytechnical University, Xi'an 710072, China

${ }^{\mathrm{b}}$ Shenzhen Key Laboratory of Special Functional Materials \& Shenzhen Engineering Laboratory for Advance Technology of ceramics, College of Materials Science and Engineering, Shenzhen University, Shenzhen 518060, PR China

${ }^{*}$ Corresponding author. Tel.: +86 755 26537459;

E-mail: zengxier@szu.edu.cn; zoujizhao@szu.edu.cn 


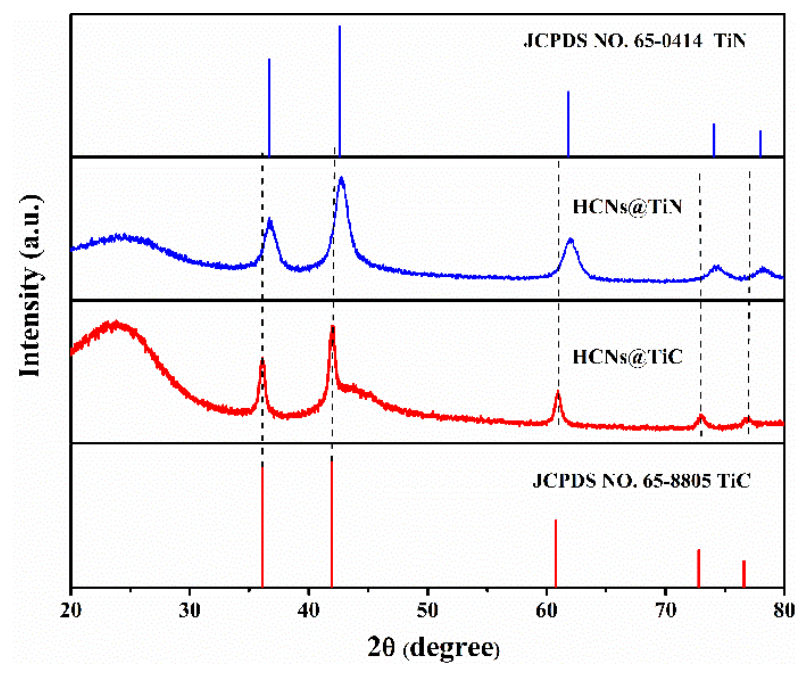

Fig. S1 XRD pattern of HCNs@TiN and HCNs@TiC

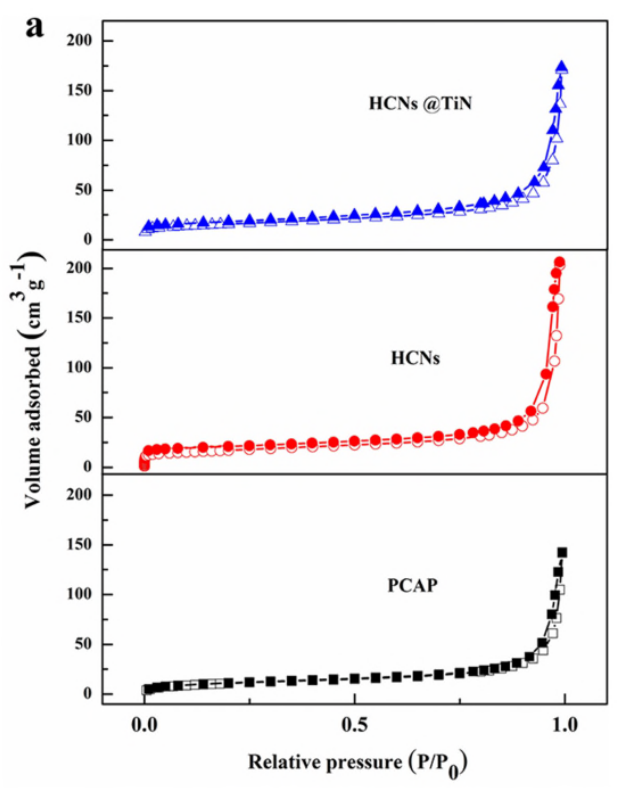

b

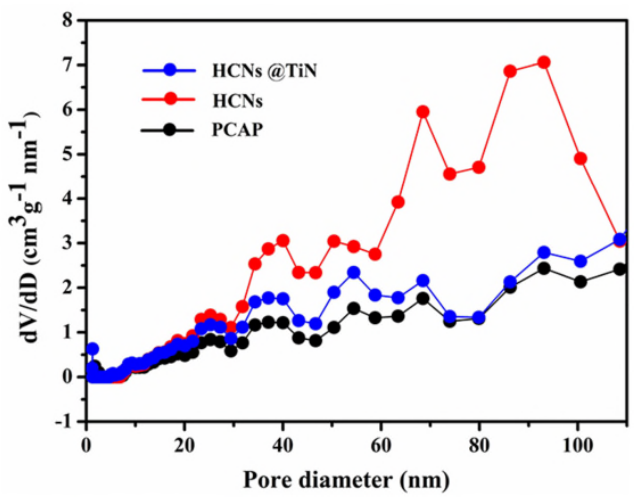

Fig. S2 (a) N2 adsorption/desorption isotherms and (b) corresponding pore size distributions of PCAP, HCNs and HCNs@TiN. 
Table S1

Surface area and pore volume of PCAP, HCNs and HCNs@TiN.

\begin{tabular}{ccc}
\hline Specimen & $\begin{array}{c}\mathrm{S}_{\mathrm{BET}} \\
\left(\mathrm{m}^{2} \mathrm{~g}^{-1}\right)\end{array}$ & $\begin{array}{c}\mathrm{V}_{\text {total }} \\
\left(\mathrm{cm}^{3} \mathrm{~g}^{-1}\right)\end{array}$ \\
\hline PACP & 39.4 & 0.16 \\
HCNs & 58.6 & 0.26 \\
HCNs@TiN & 54.9 & 0.20 \\
\hline
\end{tabular}




\section{Microwave-assisted rapid preparation of hollow carbon nanospheres@TiN nanoparticles for lithium-sulfur batteries}

Tu, Jianxin

Royal Society of Chemistry

Jianxin Tu, HeJun Li, JiZhao Zou, et al., (2018) Microwave-assisted rapid preparation of hollow carbon nanospheres@TiN nanoparticles for lithium-sulfur batteries. Dalton Transactions, Volume 47, December 2018, pp. 16909-16917

https://doi.org/10.1039/C8DT04095H

Downloaded from Cranfield Library Services E-Repository 